\title{
Introducción de la evaluación del trabajo en equipo en la asignatura Vibraciones Mecánicas
}

Andrés Rovira ${ }^{a}$, Casto Bolumar ${ }^{b}$, Natalia Lajara-Camilleri ${ }^{c}$ y Alexis J. Bañón-Gomis ${ }^{d}$ ${ }^{a}$ CIIM. Dpto. de Ingeniería Mecánica y de Materiales. Universitat Politècnica de València. arovira@mcm.upv.es, bDpto. de Ingeniería Mecánica y de Materiales. Universitat Politècnica de València. casbola@mcm.upv.es, 'CEGEA. Dpto. de Economía y Ciencias Sociales. Universitat Politècnica de València. nalade@cegea.upv.es y ${ }^{\mathrm{d}}$ Dpto. de Organización de Empresas. Universitat Politècnica de València. albaogo@upvnet.upv.es.

\begin{abstract}
With the implementation of the curriculum of the European Higher Education Area (EHEA ) a new training paradigm, based on competencies, is adopted.

This article discusses the implementation of the training and assessment of the teamwork and leadership competency in pilot groups of the Mechanical Vibrations course of the degree in Mechanical Engineering.

For the training of the competency in classroom it was used a good practice guide, which seeks to influence and give pause to students in the interpersonal dimension that involves teamwork. This document provides recommendations and suggestions for good management of social relations and achieve a strategy of working together.
\end{abstract}

The work presented is part of the dissemination of the results of an innovation project carried out in this course 2014-2015.

Keywords: EHEA, competency assessment, UPV transversal competencies, teamwork and leadership, good practice guide

\footnotetext{
Resumen

Con la implantación de los planes de estudio del Espacio Europeo de Educación Superior (EEES) se adopta un paradigma de formación en competencias.

En este artículo se presenta la implementación de la formación y evaluación de la competencia transversal de trabajo en equipo en grupos piloto de la asignatura Vibraciones Mecánicas del plan de estudios de Grado en Ingeniería Mecánica.
} 
Para el trabajo de la competencia en el aula se ha utilizado una guía de buenas prácticas, que busca incidir y hacer reflexionar a los alumnos en la dimensión interpersonal que implica el trabajar en equipo. Este documento presenta recomendaciones y consejos para conseguir una buena gestión de las relaciones sociales y lograr una estrategia de trabajo en común.

Para la evaluación, se ha considerado tanto el producto como el proceso seguido. Por un lado, los profesores han calificado el trabajo realizado por los alumnos; por otro lado, los alumnos han evaluado su propia actuación en el grupo a través de un cuestionario de autoevaluación.

Este trabajo expuesto forma parte de la difusión de los resultados obtenidos en un Proyecto de Innovación y Mejora Educativa (PIME) llevado a cabo en el presente curso 2014-2015.

Palabras clave: EEES, evaluación de competencias, competencias transversales UPV, trabajo en equipo y liderazgo, guía de buenas prácticas

\section{Introducción}

El presente artículo describe la experiencia realizada en la asignatura Vibraciones Mecánicas en relación al entrenamiento (o trabajo) de la competencia de trabajo en equipo y liderazgo. La asignatura forma parte del cuarto curso de la titulación de Graduado en Ingeniería Mecánica impartida en la Escuela Técnica Superior de Ingeniería del Diseño (ETSID) de la Universitat Politècnica de València (UPV).

Esta experiencia se ha llevado a cabo tomando unos grupos pilotos en la asignatura y en uno de los trabajos académicos que deben realizar los alumnos. En este trabajo académico se ha evaluado tanto el producto realizado por los alumnos, como el proceso seguido por cada uno de los componentes del grupo.

La innovación introducida en esta experiencia es doble:

- En primer lugar, se ha utilizado una guía de buenas prácticas para trabajar la competencia de trabajo en equipo. En este guía se incide en la importancia de las relaciones interpersonales en el trabajo en equipo y se incluyen unas recomendaciones y pautas de comportamiento, formuladas en positivo.

- En segundo lugar, la evaluación del trabajo en equipo incluye no sólo la evaluación del producto presentado, sino también la evaluación del proceso realizado. Esta evaluación sólo puede llevarse a cabo por parte de los componentes del grupo. 
En relación a este segundo punto, los alumnos comentaron que, aunque habían hecho previamente trabajos en equipo, no se les había evaluado su actuación dentro del grupo.

\section{Objetivos}

Los objetivos de este trabajo son dos:

- Presentar la experiencia realizada en la asignatura vibraciones mecánicas así como los recursos utilizados.

- Presentar los resultados de la autoevaluación realizada por los alumnos en relación al trabajo en equipo.

\section{Desarrollo de la innovación}

En este apartado en primer lugar se describe la competencia de trabajo en equipo y liderazgo. Posteriormente, se presenta cómo se ha trabajado la competencia en el aula, a través de una guía de buenas prácticas. Finalmente, se trata la evaluación del trabajo realizado y se presenta la rúbrica empleada para la evaluación del trabajo en equipo.

\subsection{La competencia de trabajo en equipo y liderazgo}

Villa y Poblete (2007) definen la competencia de "trabajo en equipo y liderazgo" como la integración y colaboración de forma activa en la consecuencia de objetivos comunes con otras personas, áreas y organizaciones, influyendo sobre las personas y/o grupos, anticipándose al futuro y contribuyendo al desarrollo personal y profesional. De acuerdo con estos autores se trata de una de las competencias clave, pues suponen pensar analítica y sistemáticamente, administrar el tiempo de trabajo, participar en toma de decisiones y en gestión de objetivos y proyectos.

Por otro lado, la UPV define la competencia transversal de "trabajo en equipo y liderazgo" como trabajar y liderar equipos de forma efectiva para la consecución de objetivos comunes, contribuyendo al desarrollo personal y profesional de estos equipos. Esta competencia constituye la número 6 de las 13 competencias transversales definidas en la UPV.

Estas dos definiciones permiten extraer unos objetivos comunes que, en nuestro caso, serán la realización de un trabajo académico, trabajar en equipo y contribuir a su desarrollo. Este planteamiento se hace en base a que se trata de una competencia interpersonal y a las tareas involucradas en el propio trabajo en equipo, que son: pensar analítica y sistemáticamente, administrar el tiempo, tomar decisiones y gestionar proyectos. El entrenamiento de esta competencia en la asignatura y su posterior evaluación deben considerar todos estos aspectos.

\section{(cc) EY-NC-ND 2015, Universitat Politècnica de València}




\subsection{Guía de buenas prácticas}

La naturaleza interpersonal de esta competencia puede desarrollarse mejor mediante el marco de actuación que representa la guía de buenas prácticas. Ésta se desarrolló por el presente grupo de investigación el curso académico 2013-2014 en el marco de un Proyecto de Innovación y Mejora Educativa (PIME) y se publicó en Bañón-Gomis et al. (2014a). La guía de buenas prácticas se muestra en el Anexo 1.

El objetivo de la guía esincidir en la dimensión interpersonal del trabajo en equipo. Para ello se introducen aspectos como la composición del equipo y la organización del trabajo. Asímismo se incluye un decálogo con recomendaciones para la gestión del trabajo en equipo en las distintas tareas que lo componen (pensar analítica y sistemáticamente, administrar el tiempo, tomar decisiones y gestionar proyectos), incluida la resolución de conflictos.

En la experiencia descrita, esta guía de buenas prácticas fue especialmente útil para dar a conocer esta dimensión interpersonal. Además, al tratarse de una guía de buenas prácticas ofrece un planteamiento positivo y optimista, por lo que tuvo muy buena acogida entre los alumnos.

\subsection{Evaluación}

La evaluación del trabajo académico se hizo de dos formas: por un lado se calificó el producto del trabajo, es decir, el propio informe, relacionado con la competencia transversal de comunicación efectiva a nivel escrito; por otro lado, se evaluó la actuación en el grupo, relacionada con el trabajo en equipo.

La calificación del producto, es decir, el informe o trabajo académico entregado, se hizo en función de una lista de control que incluía los siguientes ítems: organización, presentación y estructura (25\%); descripción del sistema estudiado y justificación de la solución adoptada (25\%), presentación de las señales de excitación y respuesta (25\%) y conclusiones (25\%). Según la definición de competencias transversales UPV (UPV 2013), esta calificación hace referencia tanto a la competencia transversal de comunicación efectiva, en relación a la elaboración de informes técnicos, como a la competencia instrumental específica, en relación a los propios contenidos que debe tener el informe.

Para la evaluación del trabajo en equipo, desde un punto de vista práctico, aparece la dificultad de obtener evidencias directas de la participación de cada uno de los miembros en el trabajo en equipo. Esta característica obliga al uso de alternativas a las técnicas tradicionales en las que el profesor se encarga del $100 \%$ de la evaluación de sus alumnos. Por tanto, para la evaluación del trabajo en equipo es necesario contar con la participación de los 
miembros del grupo, es decir, los alumnos. De esta forma, se pueden plantear dos estrategias: la evaluación entre iguales o la autoevaluación.

La evaluación entre iguales se hace de tal forma que cada miembro del equipo emite un informe sobre cada uno de sus compañeros (en "abanico"); por tanto el resultado para cada uno de los miembros del grupo tendría en cuenta lo que "ha dicho" cada uno de sus compañeros. La autoevaluación se obtiene a partir de una reflexión que realiza cada miembro del equipo a partir de su actuación en grupo. Puesto que el objetivo de esta experiencia es estimular la reflexión de cada alumno en la dimensión interpersonal del trabajo en equipo, se ha optado por la segunda opción. La evaluación del trabajo en equipo se ha realizado a través de un cuestionario de autoevaluación, basado en una tabla de evaluación o rúbrica, que se describe en la próxima sección.

Puesto que la competencia de trabajo en equipo y liderazgo no forma parte de las competencias trabajadas en la asignatura según su guía docente, y de que sólo se ha aplicado esta experiencia a un par de grupos piloto de la asignatura, la evaluación del trabajo en equipo no se tuvo en cuenta en la calificación final de la asignatura. En cambio, esta evaluación sí que es útil para definir el "perfil competencial" de los estudiantes en asignaturas tomadas como "puntos de control" en cada titulación, y sirve como una experiencia piloto para la obtención de evidencias que acrediten el grado de aprendizaje de competencias transversales.

\subsection{Rúbrica para la evaluación del trabajo en equipo}

El uso de tablas de evaluación o rúbricas en la evaluación por iguales o la autoevaluación mejora la implicación de los estudiantes y sus resultados. El empleo de estas herramientas por parte de los alumnos constituye un ejercicio de responsabilidad y autocrítica que contribuye a su desarrollo personal.

En la literatura existen numerosos artículos relacionados con la evaluación del trabajo en equipo. En el PIME en el que se enmarca la experiencia se tomó como base la rúbrica desarrollada por Chica (2011) ya que presenta una estructura muy clara e inteligible, además de cubrir los distintos aspectos que definen el trabajo en equipo. Esta rúbrica considera los siguientes cinco ítems: contribución al grupo, actitud, responsabilidad, asistencia a reuniones y resolución de conflictos. En Bañón-Gomis el al. (2014b) se propone añadir un ítem que incluya la organización del grupo en cuanto a compartir recursos, cooperar y conseguir los objetivos del grupo. La Tabla 1 muestra un esquema de la rúbrica utilizada, con cuatro niveles de desempeño, ordenados de mejor a mayor. La descripción de cada uno de los niveles de desempeño para cada uno de los ítems se incluye en el Anexo 2.

Esta rúbrica se implementó en un cuestionario de Google Drive, accesible a través de la URL http://goo.gl/YB6s96, que permitió obtener los datos de forma automática.

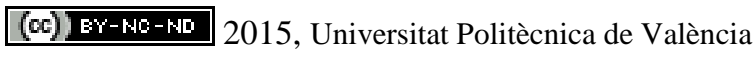

Congreso In-Red (2015) 
Tras la entrega del informe (trabajo académico) por parte de los alumnos, el profesor correspondiente envió una invitación, a cada uno de los miembros del grupo, para responder el cuestionario.

Tabla 1. Esquema de la rúbrica empleada para evaluar el trabajo en equipo.

\begin{tabular}{|c|c|c|c|c|}
\hline \multirow[b]{2}{*}{ Ítem } & \multicolumn{4}{|c|}{ Nivel de desempeño } \\
\hline & Pobre & Escaso & Bueno & Excelente \\
\hline Contribución al grupo & a.1 & a. 2 & a.3 & a. 4 \\
\hline Actitud & b.1 & b. 2 & b.3 & b. 4 \\
\hline Responsabilidad & c. 1 & c. 2 & с.3 & c. 4 \\
\hline $\begin{array}{c}\text { Asistencia a reuniones } \\
\text { y puntualidad }\end{array}$ & d.1 & d. 2 & d.3 & d. 4 \\
\hline $\begin{array}{c}\text { Organización del } \\
\text { grupo }\end{array}$ & e.1 & e. 2 & e.3 & e. 4 \\
\hline $\begin{array}{l}\text { Resolución de } \\
\text { conflictos }\end{array}$ & f.1 & f. 2 & f.3 & f. 4 \\
\hline
\end{tabular}

\section{Resultados}

Los resultados obtenidos en la evaluación de los distintos aspectos del trabajo en equipo provienen del cuestionario de autoevaluación respondido por los alumnos, basado en la rúbrica de la sección 3.4. Como se ha comentado anteriormente, la evaluación del tabajo en equipo no tenía influencia en la nota final; por tanto, para premiar la participación de los alumnos, se les envío un informe de realimentación a cada uno de ellos. Cabe destacar que la totalidad de los alumnos respondieron a dicho cuestionario.

Tras recibir las respuestas de los alumnos del cuestionario de autoevaluación, el profesor responsable realizó un informe de realimentación individualizado para cada uno de los alumnos. Este informe contenía las respuestas que el propio alumno había dado, junto a la respuesta promedio de sus compañeros.

La Fig. 1 muestra un ejemplo de un informe de autoevaluación enviado a los alumnos. El objetivo de este informe es hacer reflexionar a cada alumno sobre su actuación en el trabajo en equipo. La respuesta promedio se incluye con el fin de que puedan contextualizar el perfil obtenido por cada uno. 


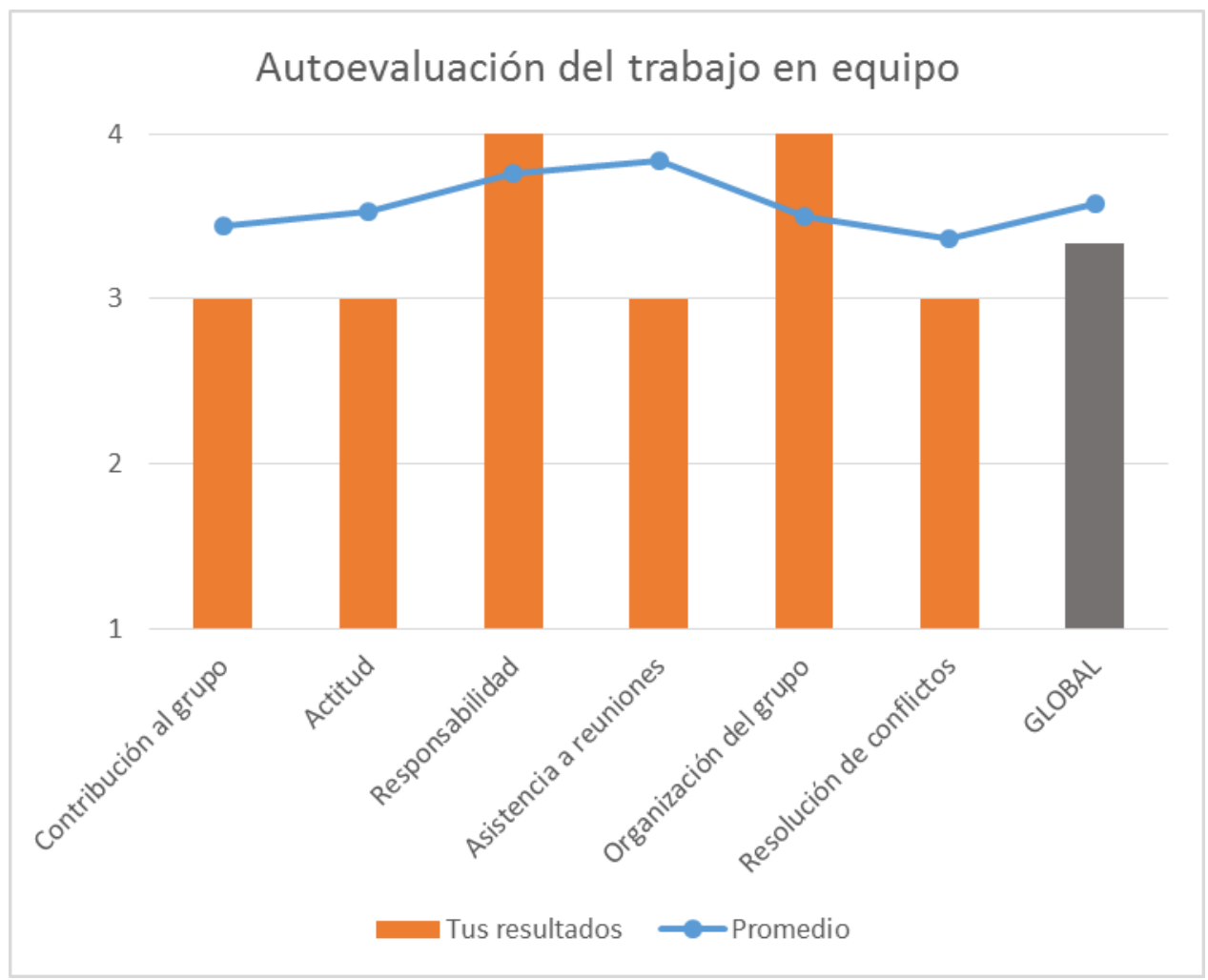

Fig. 1 Ejemplo de un informe de realimentación enviado a un alumno.

En general, los ítems que han obtenido una mayor valoración son la "asistencia a reuniones”, -entendido como el cuidado y diligencia en concurrir a las reuniones - y la "responsabilidad" -realización de tareas asignadas por el grupo dentro de los plazos requeridos-. Por otro lado, los ítems que han obtenido una puntuación más baja son la "resolución de conflictos" entendido como la capacidad de diagnosticar, afrontar y resolver conflictos interpersonales con prontitud y profundidad, sin dañar la relación personal- y la “contribución al grupo”participación activa en los espacios de encuentro del equipo-. No obstante todos los ítems tienen una valoración entre buena (3) y excelente (4).

Analizando en más profundidad los resultados comentados en el párrafo anterior, sería interesante tomar aquéllos que han obtenido una valoración más baja en la autoevaluación realizada por los alumnos y trabajarlos en asignaturas del mismo plan de estudios.

(cc) EY-NC-ND 2015, Universitat Politècnica de València

Congreso In-Red (2015) 


\section{Conclusiones}

Las principales conclusiones de este trabajo son las siguientes:

- La implantación del sistema de enseñanza basado en competencias, implica el trabajo y evaluación de éstas.

- Considerando la competencia transversal de trabajo en equipo y liderazgo se debe considerar la dimensión de las relaciones interpersonales y no sólo el "producto" obtenido en dicho trabajo.

- Para el entrenamiento de la competencia de trabajo en equipo y liderazgo se ha usado como referencia una guía de buenas prácticas que incluye recomendaciones y consejos, formados en positivo, para conseguir unos objetivos comunes. Esta actividad fue muy bien acogida por los alumnos.

- Para la evaluación del trabajo en equipo aparece la dificultad de obtener evidencias directas de la participación de cada uno de los miembros; por tanto, es necesario contar con la participación de los miembros del grupo, es decir, los alumnos.

- En la experiencia descrita se ha optado por un sistema de autoevaluación, con el fin de hacer reflexionar a los alumnos sobre las distintas cuestiones que implica el trabajo en equipo.

- Los resultados obtenidos revelan como aspectos más autoevaluados la "asistencia a reuniones” y la "responsabilidad” y en cuanto a los menos evaluados, la "resolución de conflictos” y la “contribución al grupo”.

La evaluación del trabajo en equipo se ha mostrado útil para determinar el "perfil competencial” de los estudiantes y para obtener evidencias del grado de aprendizaje de dicha competencia transversal. Asímismo, se considera importante realizar una especial mención a la importancia de la evaluación con independencia de que sea finalmente calificada o no. La mera evaluación no sólo sirve para poder diagnosticar la situación de cada alumno sino también para poder ayudarle a superarse mediante técnicas como el coaching o el mentoring.

\section{Agradecimientos}

Este trabajo ha sido financiado por el Vicerrectorado de Estudios, Calidad y Acreditación y la Escuela Técnica Superior de Ingeniería del Diseño (ETSID) de la Universitat Politècnica de València (UPV) a través de la convocatoria de ayudas para PIME 2014-2015, con el proyecto código B019. 


\section{Referencias}

BAÑÓN-GOMIS, A.J. et al. (2014a). "Experiencias docentes en la UPV en torno a la competencia transversal "trabajo en equipo y liderazgo". En IN-RED. Jornadas de Innovación Educativa y de Docencia en Red de la Universitat Politècnica de València. Valencia 15-16 de julio. Disponible en: $<$ http://riunet.upv.es/handle/10251/40404> [Consulta 25 de mayo de 2015]

BAÑÓN-GOMIS, A.J. et al. (2014b). "Three-Dimensionality in competencies: the inclusion of ethics in the generic competency of teamwork and leadership” en Peris-Ortiz, M, Merigó.-Lindahl, J.M. Sustainable learning in Higher Education. Developing competencies for the Global Marketplace. New York: Springer. ISBN: 9783319108032. p.p. 143-155

CHICA, E. (2011). Una propuesta de evaluación para el trabajo en grupo mediante rúbrica. Escuela abierta, 14, 67-81

UPV (2013). “Dimensiones Competenciales UPV”, Borrador de trabajo, Valencia

VILLA, A., POBLETE, M. (2007). Aprendizaje basado en competencias. Una propuesta para la evaluación de las competencias genéricas. Ediciones Mensaje. Universidad de Deusto. Bilbao. 
Introducción de la evaluación del trabajo en equipo en la asignatura Vibraciones Mecánicas

\section{Anexo 1. Guía de buenas prácticas para el trabajo en equipo.}

\section{Introducción}

Trabajar en equipo es una forma de lograr metas que por nosotros mismos, individualmente, no podríamos alcanzar. Por supuesto, conseguiríamos hacer el mismo trabajo (o incluso mejor) pero con una inversión de recursos -fundamentalmente tiempo- mucho mayor.

El problema surge cuando hay ineficiencias en el grupo, eso produce desgaste en sus miembros y hace que los resultados sean inferiores a los deseados o bien que los recursos empleados sean mayores que los planificados inicialmente.

Es importante desarrollar una metodología de trabajo en equipo adecuada porque vivimos en sociedad. Tanto en el ámbito educativo como profesional y a nivel personal debemos saber adoptar las estrategias necesarias para tener éxito tanto si desarrollamos un trabajo individual como si lo hacemos en colaboración con otras personas.

En esta guía queremos daros algunos consejos y recomendaciones a tener en cuenta de cara a los trabajos en equipo.

\section{¿Son importantes las personas con las que formo el equipo?}

Son fundamentales. Tienen más probabilidades de éxito los equipos formados por personas que se complementan. Por ello, tanto si queremos montar una empresa como si vamos a preparar una presentación para una asignatura, tenemos que meditar bien con quién nos asociamos. Guiarse únicamente por la amistad es un error, hay que tener en cuenta todos los factores y aprender a trabajar con cualquier persona (es un tópico pero lo cierto es que en la vida profesional en la mayoría de las ocasiones no podremos elegir a nuestros compañeros).

¿Qué debo preparar antes de una primera reunión del equipo?

Cuando empezamos un trabajo tenemos que tener claros los resultados que queremos conseguir con el trabajo, nuestras capacidades y nuestra disponibilidad. Hay que ser honestos y en la primera puesta en común acordar el nivel de compromiso y los objetivos del grupo. Es una especie de "contrato" entre los miembros del grupo.

\section{¿Cómo organizar el trabajo en equipo?}

Los equipos toman decisiones y organizan tareas en las reuniones. Para ello hay que preparar con antelación las sesiones y llevar el material necesario. No todo el mundo tiene la misma disponibilidad de tiempo por lo que hay que intentar que las reuniones se hagan en el menor tiempo posible y estableciendo sólo aquellas que sean necesarias. Haremos un orden del día con los temas a tratar y tras la reunión haremos un acta sintética que contenga toda la información relevante (asistentes, temas tratados, decisiones adoptadas, tareas encargadas y responsables asignados). El acta debe permitir a un miembro que no ha asistido a la reunión tener una idea de lo hablado y acordado.

Recomendaciones para el día a día del trabajo en equipo.

1. Cuando valores el trabajo de un compañero sé concreto. Especialmente si tu crítica es para mejorar el resultado debes decir exactamente a qué te refieres. Obviamente hay que desterrar cualquier referencia personal.

\begin{tabular}{|l|l|}
\hline En lugar de... & Estaría mejor decir... \\
\hline Esto está fatal, no lo podemos entregar así & $\begin{array}{l}\text { Deberíamos hacer un índice y darle formato antes de } \\
\text { entregarlo }\end{array}$ \\
\hline No me gusta el PowerPoint & $\begin{array}{l}\text { Creo que deberíamos cambiar la estructura del } \\
\text { PowerPoint/poner ejemplos/... }\end{array}$ \\
\hline
\end{tabular}

2. Equivocarse está permitido, nos pasa a todos, tanto cuando trabajamos individualmente como en equipo. Hay que aceptar los fallos de los demás y no esconder los propios. Buscadle el valor positivo y la oportunidad que suponen.

3. Hay que ser realista con lo que asumimos que podemos hacer y responsable con los compromisos que adoptamos. Si no se va a poder hacer una tarea, es mejor decirlo y buscar la forma de compensarlo en otro momento. La falta de cumplimiento, aparte de retrasar, genera malestar e incomodidad en los equipos. La 
responsabilidad se plasma también en la puntualidad. Sed puntuales tanto en reuniones como en las entregas de tareas. Quien llega tarde a la reunión hace perder el tiempo a todos los que llegaron a la hora convenida, es una falta de respeto.

4. Desglosad el trabajo a realizar en tareas, asignadles responsables y poned fechas límite, es la forma de organizarse efectivamente.

5. Aprovechad la tecnología: usad Google Docs, Dropbox, etc para compartir archivos. En documentos de Office podéis utilizar el Control de cambios y Doodle es útil a la hora de encontrar huecos disponibles para hacer una reunión.

6. Los conflictos -que son inevitables- conviene atajarlos cuanto antes para que no se conviertan en verdaderos problemas. Hablad y negociad de forma que todos ganéis al final, en caso contrario no estará cerrado el tema.

7. Trabajando en equipo no tiene sentido la actitud de "yo lo sé todo y lo hago mejor que nadie". Escuchad a los demás atentamente, normalmente la mejor idea es la que se construye entre todos. Además no podemos imponer nuestros estándares de calidad sino que tendremos que asumir los que sean acordados por el grupo.

8. Favoreced la unidad y la participación de todos los miembros del equipo. Si hay alguien que tiene problemas para asumir algún aspecto del trabajo, el equipo debe ayudarle a superar la dificultad, bien sea asignándole otra tarea o bien echándole una mano.

9. Celebrad también los éxitos y compartidlos. Lo que consigue el equipo es mérito de todos y hay que reconocerlo.

10. Confía en tus compañeros, déjales hacer lo suyo y recurre a ellos en caso de necesitar ayuda. Si no confías en ellos, estarás constantemente desviando tu atención para vigilar las tareas de los demás. Con la confianza de que el trabajo está bien organizado y distribuido, podrás centrarte en tu parte y al final todo encajará a la perfección. La confianza también quiere decir que si necesitas ayuda, puedes pedirla.

\section{Anexo 2. Contenido de la rúbrica de la Tabla 1.}

¿Cómo valorarías tu contribución al grupo? Participación de forma activa en los espacios de encuentro del equipo (compartiendo información, conocimientos y experiencias).

- Pobre (a.1) No he ofrecido ideas para realizar el trabajo ni se me han ocurrido sugerencias para mejorarlo. Soy consciente de que en ocasiones mi actitud ha dificultado que se alcancen los objetivos del grupo.

- Escaso (a.2) He ofrecido alguna idea para hacer el trabajo pero una vez decidido el tema la verdad es que me he desentendido un poco y no he aportado sugerencias para mejorarlo. Sí que he aceptado propuestas de otros miembros del equipo para alcanzar los objetivos del grupo.

- Bueno (a.3.) He ofrecido ideas para realizar el trabajo y alguna sugerencia para mejorarlo. Me he esforzado en alcanzar los objetivos del grupo.

- $\quad$ Excelente (a.4) He ofrecido muchas ideas para realizar el trabajado y me he implicado activamente en su mejora. Me he esforzado en conseguir los objetivos del grupo.

¿Cómo valorarías tu actitud? Se trata de valorar si se toman en cuenta los puntos de vista de los demás y se da una retroalimentación de forma constructiva.

- Pobre (b.1) Me cuesta escuchar y aceptar las ideas de los demás. Mi actitud no ayuda a mantener la unidad en el grupo.

- Escaso (b.2) A veces escucho las ideas y trato de integrarlas en mis propuestas aunque no siempre lo hago. La unión del grupo creo que no es importante ni debe preocuparnos.

- Bueno (b.3) Me gusta escuchar las ideas de los demás y trato de compartirlas pero reconozco que no siempre las tengo en cuenta para reformular mis aportaciones. Trato de colaborar para manterner la unidad en el grupo.

- $\quad$ Excelente (b.4) Siempre escucho e integro las ideas aportadas para hallar soluciones que aglutinen las mejores aportaciones. Trato activamente de mantener la unión en el grupo.

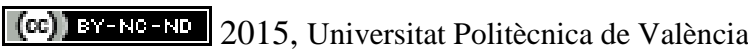

Congreso In-Red (2015) 
Introducción de la evaluación del trabajo en equipo en la asignatura Vibraciones Mecánicas

¿Cómo calificarías tu responsabilidad? Es la realización de las tareas asignadas por el grupo dentro de los plazos requeridos.

- Pobre (c.1) No he entregado el trabajo a tiempo y el grupo ha tenido que modificar su planificación en consecuencia.

- Escaso (c.2) Me he retrasado en alguna ocasión y eso ha influido de alguna manera en el trabajo del grupo.

- Bueno (c.3) He podido retrasarme ligeramente pero ha sido de forma puntual y no ha afectado al trabajo del grupo.

- $\quad$ Excelente (c.4) He entregado mi parte siempre en plazo.

Valoración de la asistencia a reuniones y puntualidad. Es el cuidado y diligencia en concurrir a las reuniones.

- Pobre (d.1) He asistido a menos del $60 \%$ de las reuniones y he llegado tarde a muchas de ellas.

- Escaso (d.2) He asistido a un 60-75\% de las reuniones y no siempre he sido puntual.

- Bueno (d.3) He asistido a casi todas las reuniones (entre el 75 y el $90 \%$ ) y siempre he sido puntual.

- $\quad$ Excelente (d.4) He ido a todas las reuniones y he sido siempre puntual.

¿Cómo calificarías tu aportación a la organización del grupo? Se trata de la colaboración en la definición, organización y distribución de las tareas del grupo.

- Pobre (e.1) No he compartido recursos disponibles con otros compañeros. Han primado mis objetivos individuales frente a los grupales y me ha costado colaborar con otros compañeros.

- Escaso (e.2) En algunas ocasiones he compartido recursos con compañeros. Me gusta pensar como un equipo porque mis objetivos pesan más para mí pero consigo cooperar.

- Bueno (e.3) Actúo como miembro del equipo y no me centro sólo en mis propios objetivos. Hago un esfuerzo (y consigo) cooperar con otros compañeros y compartir resultados aunque sé que no tan activamente como debería.

- Excelente (e.4) Siempre comparto mis recursos y me centro en el equipo en lugar de pensar en mis objetivos. Colaboro activamente con el resto de mis compañeros.

¿Cuál es tu actitud en la resolución de conflictos? Es la capacidad de diagnosticar, afrontar y resolver conflictos interpersonales con prontitud y profundidad, sin dañar la relación personal.

- Pobre (f.1) Cuando estoy involucrado en situaciones de conflicto o desacuerdo me cuesta escuchar a los demás 0 aceptar sugerencias. Cuando el conflicto es entre otros miembros creo que es mejor no meterse y que se solucione el problema sin mí.

- Escaso (f.2) En situaciones de conflicto o desacuerdo, pocas veces escucho otras opiniones 0 acepto sugerencias. No suelo proponer alternativas para el consenso pero las acepto.

- Bueno (f.3) En situaciones de conflicto o desacuerdo, casi siempre escucho otras opiniones o acepto sugerencias. A veces propongo alternativas para el consenso pero las acepto.

- Excelente (f.4) En situaciones de conflicto o desacuerdo, siempre escucho otras opiniones 0 acepto sugerencias. Siempre propongo alternativas para el consenso y las acepto con facilidad. 